EPJ manuscript No.

(will be inserted by the editor)

\title{
Dynamical Coupled-Channels Analysis at EBAC
}

\author{
T. -S. H. Lee Le, $^{1,2}$ \\ 1 Physics Division, Argonne National Laboratory, Argonne, IL 60439 \\ 2 Excited Baryon Analysis Center (EBAC), Thomas Jefferson National Accelerator Facility, Newport News, Va. 22901
}

Received: date / Revised version: date

Abstract. The status of the dynamical coupled-channels analysis at EBAC is reported.

PACS. 1 3.75.Gx, 13.60.Le, 14.20.Gk

\section{Introduction}

In this contribution, we report on the dynamical coupledchannels analysis being pursued at the Excited Baryon Analysis Center (EBAC) of Jefferson Laboratory. EBAC was established in January, 2006. Its objective is to extract the parameters associated with the excited states $\left(N^{*}\right)$ of the nucleon from the world data of meson production reactions, and to also develop theoretical interpretations of the extracted $N^{*}$ parameters.

Since $N^{*}$ states are unstable, their structure must couple with the reaction channels in the meson production reactions. To determine correctly the spectrum of $N^{*}$ states, an analysis of the meson production data must account for the coupled-channels unitary condition. The extracted $N^{*}$ parameters can be interpreted correctly only when the reaction mechanisms in the short-range region where we want to map out the $N^{*}$ structure have been accounted for. To meet these two crucial requirements, the dynamical coupled-channels model developed in Ref.[1] is being applied at EBAC to analyze the data of $\pi, \pi \pi, \eta, K$, and $\omega$ production. It involves sloving the following coupled integral equations

$$
\begin{aligned}
& T_{\alpha, \beta}\left(p_{\alpha}, p_{\beta} ; E\right)=V_{\alpha, \beta}\left(p_{\alpha}, p \beta\right) \\
& +\sum_{\gamma} \int_{0}^{\infty} d p^{\prime} V_{\alpha, \gamma}\left(p_{\alpha}, p^{\prime}\right) G_{\gamma}\left(p^{\prime}, E\right) T_{\gamma, \beta}\left(p^{\prime}, p_{\beta}, E\right), \\
& V_{\alpha, \beta}=v_{\alpha, \beta}+\sum_{N^{*}} \frac{\Gamma_{N^{*}, \alpha}^{\dagger} \Gamma_{N^{*}, \beta}}{E-M^{*}}
\end{aligned}
$$

where $\alpha, \beta, \gamma=\gamma N, \pi N, \eta N$, and $\pi \pi N$ which has $\pi \Delta, \rho N, \sigma N$ resonant components, $G_{\gamma}(p, E)$ is the propagator of channel $\gamma, v_{\alpha, \beta}$ is defined by meson-exchange mechanisms, and $\Gamma_{N^{*}, \beta}$ is related to the quark-gluon sub-structure of $N^{*}$. At the present time, it is reasonable to interpret $\Gamma_{N^{*}, \beta}$ in terms of hadron structure calculations with effective degrees of freedom, such as the constituent quark model[2] and the model[3] based on Dyson-Schwinger Equations. In the near future, one hopes to relate $\Gamma_{N^{*}, \beta}$ to Lattice QCD (LQCD) calculations.

If we take the on-shell approximation, Eq.(1) is reduced into an algebraic form of K-matrix models[4-7]

$$
\begin{aligned}
T_{\alpha, \beta}^{k}\left(p_{\alpha}, p_{\beta}, E\right)= & \sum_{\gamma} V_{\alpha, \gamma}\left(p_{\alpha}, p_{\gamma}\right) \\
& \times\left[\delta_{\alpha, \gamma}+i \rho\left(p_{\gamma}\right) T_{\gamma, \beta}^{k}\left(p_{\gamma}, p_{\beta}, E\right)\right],
\end{aligned}
$$

where $\rho\left(p_{\gamma}\right)$ is an appropriate phase space factor. Qualitatively speaking, the use of the on-shell model based on $T_{\alpha, \beta}^{k}\left(p_{\alpha}, p_{\beta}, E\right)$ of Eq.(3) is to avoid an explicit treatment of the reaction mechanisms in the short range region where we want to map out the quark-gluon sub-structure of $N^{*}$ states. Thus the $N^{*}$ parameters extracted by using Eq.(1) can be more directly interpreted in terms of the quark-gluon sub-structure of $N^{*}$.

In section 2 , we briefly review the dynamical coupledchannels model of Ref.[1]. The recent results from EBAC are reported in section 3. Section 4 gives an outlook of EBAC.

\section{Dynamical Coupled-Channels Model}

Within the formulation of Ref.[1], Eqs.(1)-(2) are derived from the following Hamiltonian

$$
\begin{aligned}
H & =H_{0}+H_{I} \\
H_{0} & =\sum_{M B}\left[\sqrt{m_{B}^{2}+k^{2}}+\sqrt{m_{M}^{2}+p^{2}}\right] \\
H_{I} & =\Gamma_{V}+v_{22}+v_{23}+v_{33}
\end{aligned}
$$

The non-resonant interactions $v_{22}, v_{23}, v_{33}$ are derived from phenomenological Lagrangians by using a unitary transformation method $[9,10]$. The interaction $v_{22}$ is defined by the tree diagrams illustrated in Fig.2. Examples 


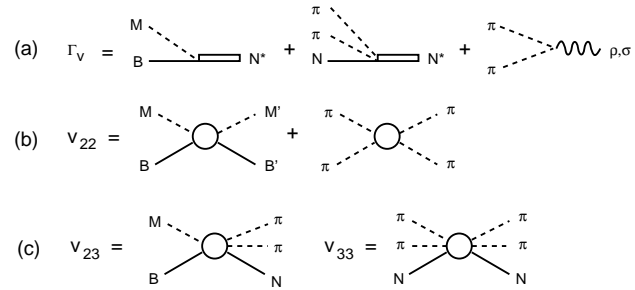

Fig. 1. Basic mechanisms of the Model Hamiltonian defined in Eqs.(4)-(6).

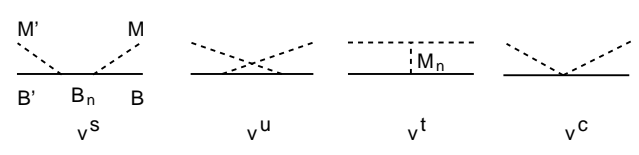

Fig. 2. Meson-baryon (MB) interaction mechanisms of $v_{2,2}$ of Eq.(6).

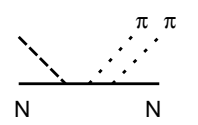

(a)

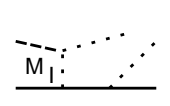

(d)

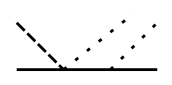

(g)

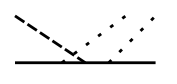

(b)

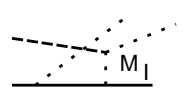

(e)

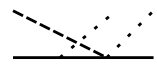

(h)

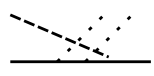

(c)

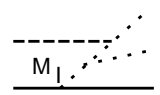

(f)
Fig. 3. Examples of non-resonant mechanisms of $v_{M N, \pi \pi N}$ with $M=\pi$ or $\gamma$ (denoted by long-dashed lines). $M_{I}$ denotes the intermediate mesons $(\pi, \rho, \omega)$.

of non-resonant mechanisms of $v_{23}$ are illustrated in Fig.3. The more complex $v_{33}$ is not included in the current analysis at EBAC.

By using the projection operator techniques, one can cast Eqs.(1)-(2) for $2 \rightarrow 2$ meson-baryon $(M B)$ transition amplitudes into the following form

$$
T_{a, b}(E)=t_{a, b}(E)+t_{a, b}^{R}(E),
$$

where $a, b, c=\gamma N, \pi N, \eta N, \pi \Delta, \rho N, \sigma N$. The non-resonant amplitude in Eq.(7) is defined by

$$
t_{a, b}(E)=V_{a, b}+\sum_{c} V_{a, c} G_{c}(E) t_{c, b}(E)
$$

where the driving term is

$$
V_{a, b}(E)=v_{a, b}+Z_{a, b}^{(E)}(E)+Z_{a, b}^{(I)}(E) .
$$

The effects due to the three-body unitarity cuts are included in $Z_{a, b}^{(E)}(E)$ and $Z_{a, b}^{(I)}(E)$, as illustrated in Figs.4-5.

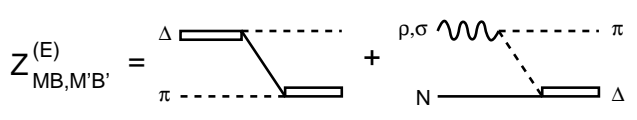

Fig. 4. One-particle-exchange interactions $Z_{\pi \Delta, \pi \Delta}^{(E)}(E)$, $Z_{\rho N, \pi \Delta}^{(E)}$ and $Z_{\sigma N, \pi \Delta}^{(E)}$ of Eq.(9).
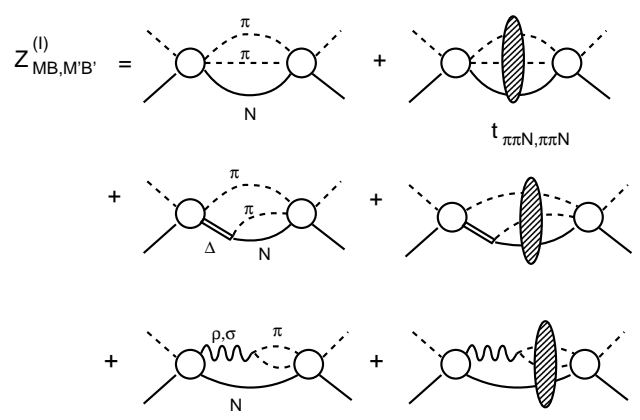

Fig. 5. Examples of mechanisms included in $Z_{M B, M^{\prime} B^{\prime}}^{(I)}$ of Eq.(9).

The resonant amplitude in Eq.(7) is defined by

$$
t_{a, b}^{R}(E)=\sum_{N_{i}^{*}, N_{j}^{*}} \bar{\Gamma}_{N_{i}^{*}, a}^{\dagger}(E)[g(E)]_{i, j} \bar{\Gamma}_{N_{j}^{*}, b}(E),
$$

where the dressed vertex is

$$
\bar{\Gamma}_{N^{*}, a}(E)=\Gamma_{N^{*}, a}+\sum_{b} \Gamma_{N^{*}, b} G_{b}(E) t_{b, a}(E),
$$

and the $N^{*}$ propagator is defined by

$$
g_{i, j}^{-1}(E)=E-M_{i}^{0} \delta_{i, j}-\sum_{a} \bar{\Gamma}_{N_{i}^{*}, a} G_{a}(E) \Gamma_{N_{j}^{*}, a}^{\dagger} .
$$

Here we emphasize that the second term of the dressed vertex Eq.(11) is a necessary consequence of the unitarity condition. This term describes the meson cloud effects on the $N^{*}$ excitations. See Ref.[8] for a more detailed discussion on this point.

To solve Eq.(8), we need to handle the singular structure of $Z_{a, b}^{(E)}$ and $Z_{a, b}^{(I)}$. Their matrix elements diverge within the moon-shapes region in Fig.6 and thus have the singular structure illustrated in Fig.7. We overcome this difficulty by using the Spline-function method developed in Refs. $[11,12]$.

\section{3 recent Results}

A computer code for solving the dynamical coupled-channels equations given in section 2 has been developed. The numerical details, in particular on the use of Spline-function methods, are explained in Ref.[1]. 


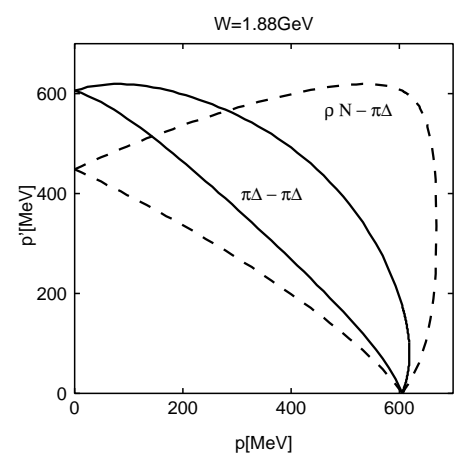

Fig. 6. Logarithmically divergent moon-shape regions of the matrix elements of $Z_{\pi \Delta, \pi \Delta}^{(E)}\left(p^{\prime}, p, E\right)$ (solid curves) and $Z_{\rho N, \pi \Delta}^{(E)}\left(p^{\prime}, p, E\right)$ (dashed curves).

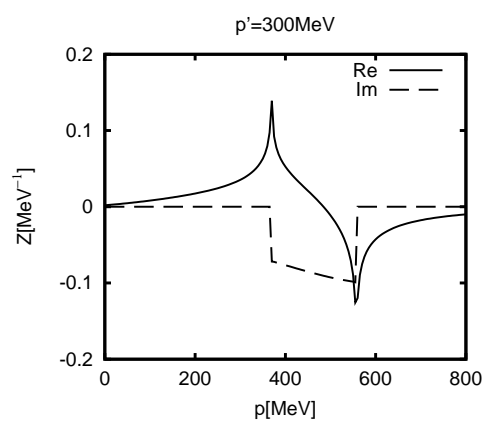

Fig. 7. Matrix elements of the one-particle-exchange term $Z_{\pi \Delta, \pi \Delta}^{(E)}\left(k, k^{\prime}, E\right)$ for $L=L^{\prime}=1, J=5 / 2, T=1 / 2$ at $k^{\prime}=300$ $\mathrm{MeV} / \mathrm{c}$ and $E=1.88 \mathrm{GeV}$.

Our first task is to determine the parameters of the hadronic interactions of the Hamiltonian defined by Eq.(6). This has been done[13] by fitting the $\pi N$ scattering data. One or two bare $N^{*}$ states in each of the $S, P, D$, and $F$ partial waves are included to generate the resonant amplitudes, defined by Eq.(10), in the fits. The parameters of the model are first determined by fitting as well as possible the empirical $\pi N$ elastic scattering amplitudes of SAID[4] up to $2 \mathrm{GeV}$. Some of our fits are shown in Figs.8-9.

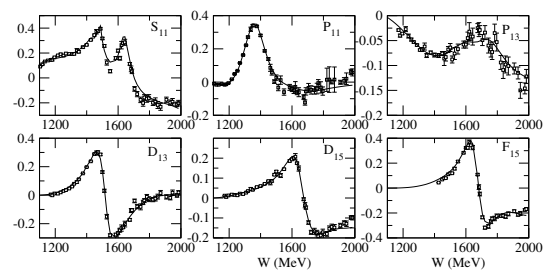

Fig. 8. Fit to the $I=\frac{1}{2} \operatorname{Re}\left(T_{\pi N, \pi N}\right)$ of $\operatorname{SAID}[4]$.

We then refine and confirm the resulting parameters by directly comparing the predicted differential cross section and target polarization asymmetry with the original data of the elastic $\pi^{ \pm} p \rightarrow \pi^{ \pm} p$ and charge-exchange

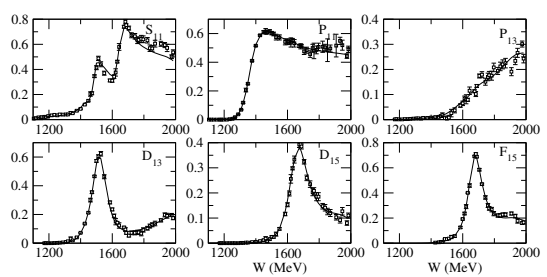

Fig. 9. Fit to the $I=\frac{1}{2} \operatorname{Im}\left(T_{\pi N, \pi N}\right)$ of $\operatorname{SAID}[4]$.

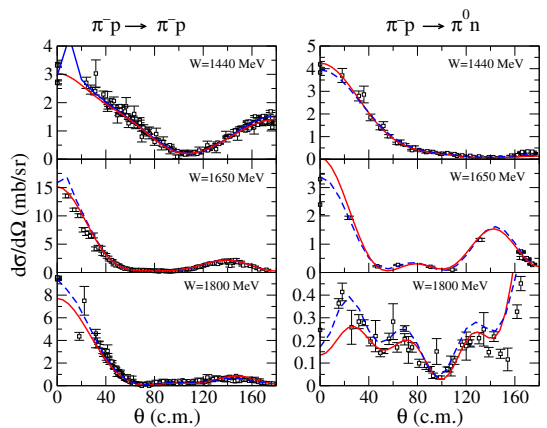

Fig. 10. Differential cross section for several different center of mass energies. Solid curves correspond to our model while blue dashed lines correspond to the SP06 solution of SAID [4]. All data have been obtained through the SAID online applications. Ref. [4].

$\pi^{-} p \rightarrow \pi^{0} n$ processes. Typical results are shown in Fig.10. The predicted total cross sections of $\pi N$ reactions are also in good agreement with the data, as shown in the left hand side of Fig.11. Our predictions of the partial total cross sections to each channel, as given in the right hand side of Fig.11, need to be refined by also fitting the $\pi N \rightarrow \pi \pi N$ data. A combined fit to both the data of $\pi N$ elastic scattering and $\pi N \rightarrow \pi \pi N$ is now being pursued at EBAC. Fig.12 is a result from this very challenging task, showing the importance of the $\pi \pi N$ cut in determining the predicted invariant mass distribution of $\pi N \rightarrow \pi \pi N$ reaction. Similar pronounced effects on $\gamma N \rightarrow \pi \pi N$ reactions have been presented in Ref.[1].

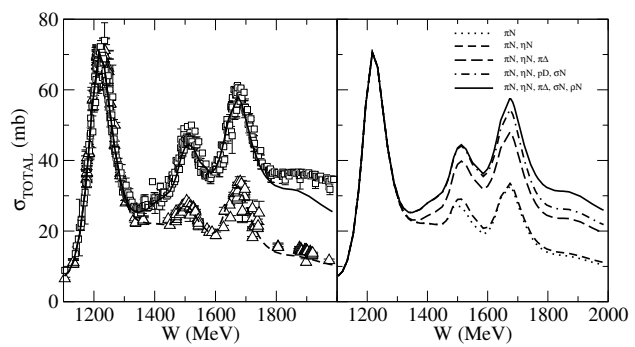

Fig. 11. Predicted $\pi^{-} p$ total cross sections.

With the hadronic parameters determined, we are now proceeding to analyzing the data of electromagnetic production of $\pi$ and $\pi \pi$. Here the only parameters to be determined are the bare $\gamma N \rightarrow N^{*}$ vertex interactions. In 


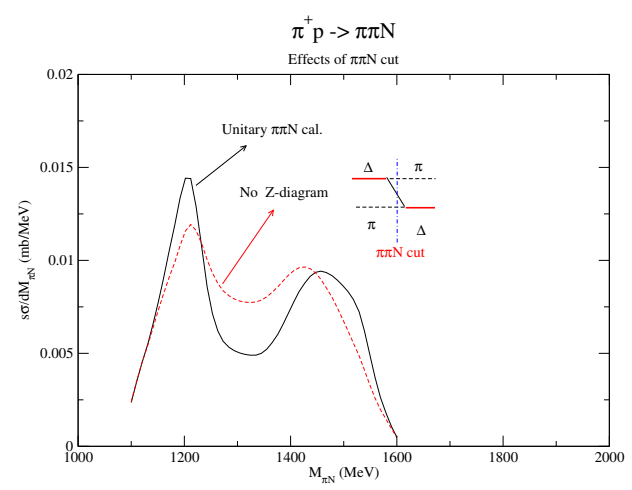

Fig. 12. The $\pi N$ invariant mass distribution of $\pi N \rightarrow \pi \pi N$ reaction at $W=1880 \mathrm{MeV}$.

Fig.13 we show the preliminary results for $\gamma p \rightarrow \pi^{+} n$. In Fig.14 we show the coupled-channels effects on the $\gamma N \rightarrow \pi N$ reactions in the $\Delta$ excitation region. More detailed results will be presented in Ref.[14].

\section{Outlook}

The dynamical coupled-channels model developed in Ref.[1] is being applied at EBAC to analyze the world data of meson production reactions. The analysis of $\pi$ and $\pi \pi$ production data is proceeding well with some encouraging preliminary results. We have also started to analyze the data of $\eta, K$, and $\omega$ production. Here the main task is to extend the model to include the interactions associated with the $K \Lambda, K \Sigma$, and $\omega N$ channels.

In parallel, we are also developing an analytical continuation method to extract the resonance poles and residues from the partial wave amplitudes predicted by the constructed dynamical coupled-channels model. Furthermore, we are investigating the extent to which the resonance poles and bare $N^{*}$ parameters extracted from our analysis can be related to the current hadron structure calculations, in particular the Lattice QCD calculations.

This work is supported by the U.S. Department of Energy, Office of Nuclear Physics Division, under contract
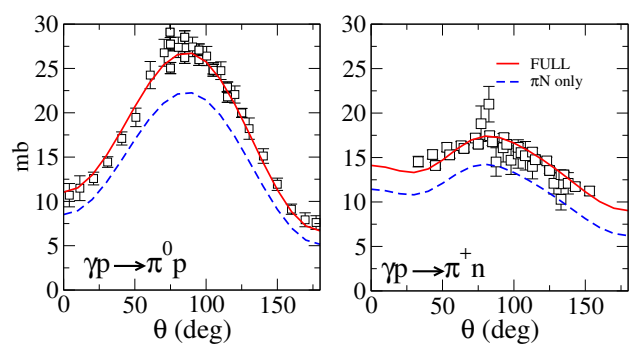

Fig. 14. Coupled-channels effects on the $\gamma N \rightarrow \pi N$ in the $\Delta$ region.

No. DE-AC02-06CH11357, and Contract No. DE-AC05060R23177 under which Jefferson Science Associates operates Jefferson Lab.

\section{References}

1. A. Matsuyama, T. Sato, T.-S. H. Lee, Phys. Rept. 439, 193 (2007).

2. See the review by S. Capstick S and W. Roberts, Prog. Part. Nucl. Phys. 45 S241 (2000).

3. See the review by P. Maris and C.D. Roberts, Int.J.Mod.Phys. E12 297(2003).

4. Arndt R, Strakovsky I, Workman R, Int. J. Mod. Phys., A18 449 (2003).

5. D. Drechsel, O. Hanstein, S. Kamalov and L. Tiator Nucl. Phys. A645, 145 (1999).

6. I. Aznauryan, Phys. Rec. C71, 01520 (2005).

7. V. Shklyar, H. Lenske, U. Mosel and G. Penner, Phys. Rev. C71, 055206 (2005).

8. B. Julia-Diaz, T.-S. H. Lee, T. Sato and L. C. Smith, Phys. Rev. C 75, 015205 (2007).

9. T. Sato and T.-S. H. Lee, Phys. Rev. C 54, 2660 (1996).

10. M. Kobayashi, T. Sato, and H. Ohtsubo, Prog. Theor. Phys. 98, 927 (1997).

11. A. Matsuyama, Phys. Lett. B152, 42 (1984).

12. A. Matsuyama and T.-S. H. Lee, Phys. Rev. C 34, 1900 (1986).

13. B. Julia-Diaz, T.-S. H. Lee, A. Matsuyama, T. Sato, to appear in Phys. Rev. C (2007).

14. B. Julia-Diaz, T.-S. H. Lee, A. Matsuyama, T. Sato, L.C. Smith, in preparation (2007).

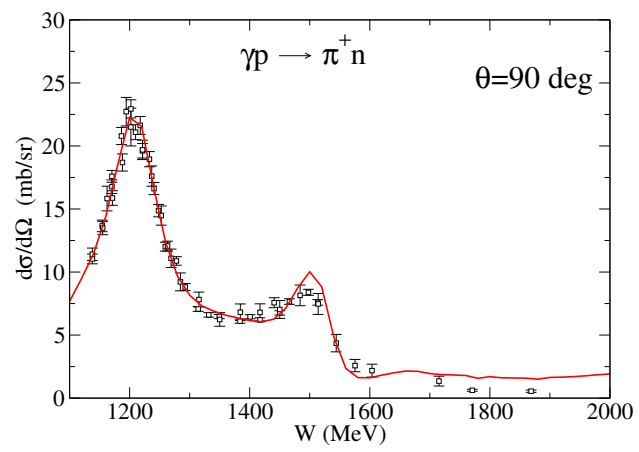

Fig. 13. Fit to the $\gamma p \rightarrow \pi^{+} p$ up to $W=2 \mathrm{GeV}$. 